This item was submitted to Loughborough's Research Repository by the author.

Items in Figshare are protected by copyright, with all rights reserved, unless otherwise indicated.

\title{
Energy-efficient resource allocation in multi-cell virtualized wireless networks
}

PLEASE CITE THE PUBLISHED VERSION

http://dx.doi.org/10.1109/ICUWB.2015.7324446

PUBLISHER

(c) IEEE

VERSION

AM (Accepted Manuscript)

LICENCE

CC BY-NC-ND 4.0

REPOSITORY RECORD

Dawadi, Rajesh, Saeedeh Parsaeefard, Mahsa Derakhshani, and Tho Le-Ngoc. 2019. "Energy-efficient

Resource Allocation in Multi-cell Virtualized Wireless Networks". figshare. https://hdl.handle.net/2134/21343. 


\title{
Energy-Efficient Resource Allocation in Multi-cell Virtualized Wireless Networks
}

\author{
Rajesh Dawadi*, Saeedeh Parsaeefard*, Mahsa Derakhshani ${ }^{\dagger}$, Tho Le-Ngoc* \\ *Department of Electrical \& Computer Engineering, McGill University, Montreal, QC, Canada \\ ${ }^{\dagger}$ Department of Electrical \& Computer Engineering, University of Toronto, Toronto, ON, Canada
}

\begin{abstract}
This paper considers the down-link transmission of an OFDMA-based multi-cell virtualized wireless network (VWN) to serve users belonging to different service providers (slices) with the quality-of-service $(\mathrm{QoS})$ requirements in terms of each slice's minimum reserved rate. In order to improve energy efficiency, we formulate a joint base station (BS) assignment, sub-carrier and power allocation problem to minimize the total transmit power of all the BSs subject to the QoS constraints. This problem is inherently a non-convex optimization problem. To tackle its computational complexity, we apply successive convex approximation (SCA) and complementary geometric programming (CGP) to convert the problem into a computationally efficient formulation and propose an iterative algorithm for solving the problem. We introduce a variable called user association factor (UAF) for jointly assigning users to both BSs and sub-carriers. Simulation results illustrate the performance enhancement of the VWN achieved through our formulation for different network scenarios.
\end{abstract}

\section{INTRODUCTION}

A new trend of virtualized wireless networks (VWNs) has recently been proposed as a promising approach to increase the spectrum and infrastructure efficiency. In a VWN, the physical wireless infrastructure is shared among multiple service providers (SPs), also called slices [1]. Each slice needs to provide a minimum reserved rate for its own users to maintain their quality of service (QoS) requirements. Consequently, an efficient resource allocation algorithm is of high importance, which has been receiving a lot of interest recently [2], [3].

For instance, in [4], interactions among slices, network operator, and users are studied by an auction. A novel admission control policy is proposed in [5] by considering the channel state information (CSI) of users in each slice to support the QoS of users. In [6], an opportunistic algorithm to allocate the resources to virtual operators is proposed. In [7], the advantages of full-duplex transmission relay in VWN is investigated. In [8], a large number of antennas is applied to a VWN to improve its total performance.

Generally, these works have focused on analyzing the resource allocation problem in a single-cell VWN scenario. However, the practical deployment of a VWN involves a multicell structure where the coverage area of a specific region will be provided by a specific set of BSs. In this paper, we investigate the resource allocation problem in a multi-cell scenario to maintain the QoS of each slice, while improving the total performance of VWN over a specific region. In this setup, it is assumed that each user of each slice can be only served by one BS and this BS is not predetermined by the distance or measuring the received signal strength. Therefore, the resource sets involve the set of BSs, sub-carriers and power for each user belonging to each slice.

Specifically, the objective of the proposed optimization problem is to minimize the total transmit power to increase the energy efficiency of VWN. Due to the downlink OFDMA limitation, one sub-carrier in each BS can only be assigned to a single user and each user can only be allocated to a single BS. We introduce a variable user association factor (UAF) to jointly determine the BS assignment and the sub-carrier allocation to a given user. From the constraints on the UAF and the inter-cell interference, the resource allocation problem is non-convex and NP-hard, which suffers from high computational complexity. To encounter this challenge, we apply the complementary geometric programming (CGP) and successive convex approximation (SCA) to propose an iterative algorithm with two steps: Step 1 derives the UAF for a given power allocation, and subsequently, for each set of UAF obtained from Step 1, Step 2 derives the optimal power allocation. In each step, we use different transformation techniques like the Arithmetic Geometric Mean Approximation (AGMA) to obtain lower-bound geometric programming (GP) formulation, which can efficiently be solved by softwares, like CVX [9].

The rest of this paper is organized as follows. In Section II, the system model and problem formulation are introduced. Section III explains the mathematical preliminaries and the proposed algorithm. Section IV presents the simulation results followed by the conclusion in Section V.

\section{SySTEM MODEL}

We consider the down-link transmission of a VWN, where the coverage of a specific area is provided by a set of BSs, i.e., $\mathcal{M}=\{1, \ldots, M\}$. The total bandwidth of $B$ $\mathrm{Hz}$ is shared between the BSs through orthogonal frequency division multiple access (OFDMA) within a set of sub-carriers $\mathcal{K}=\{1, \ldots, K\}$. It is assumed that $B_{c}=\frac{B}{K}$ is much smaller than the coherent bandwidth of the wireless channel, therefore, the CSI in each sub-carrier is flat. The set of BSs serves a set of slices, i.e., $\mathcal{G}=\{1, \ldots, G\}$, where the slice $g$ has its own set of users (denoted by $\mathcal{N}_{g}=\left\{1, \ldots, N_{g}\right\}$ ) and requests for a minimum reserved rate of $R_{g}^{\text {rsv }}$.

Let $h_{m, k, n_{g}}$ and $P_{m, k, n_{g}}$ be the CSI of the link from BS $m \in \mathcal{M}$ to user $n_{g}$ of slice $g$ on sub-carrier $k$ and allocated 
power to user $n_{g}$ of slice $g$ on sub-carrier $k$, respectively. Due to OFDMA limitation, each user is assigned to only one BS, and to avoid intra-cell interference, orthogonal sub-carrier assignment is assumed among users in a cell. Furthermore, we assume that there is no pre-allocated BS for users. The binary-valued user association factor (UAF), $\beta_{m, k, n_{g}} \in\{0,1\}$ is defined for user $n_{g}$ of slice $g$ on sub-carrier $k$ of BS $m$ as $\beta_{m, k, n_{g}}= \begin{cases}1, & \text { if BS } m \text { allocates sub-carrier } k \text { to user } n_{g} \\ 0, & \text { otherwise. }\end{cases}$ From the OFDMA exclusive sub-carrier allocation within each cell $m$, we have

C1: $\quad \sum_{g \in \mathcal{G}} \sum_{n_{g} \in \mathcal{N}_{g}} \beta_{m, k, n_{g}} \leq 1, \forall m \in \mathcal{M}, \forall k \in \mathcal{K}$.

Also, from multi-cell OFDMA limitation, we restrict the access of each user only to one BS as,

$$
\mathrm{C} 2:\left[\sum_{k \in \mathcal{K}} \beta_{m, k, n_{g}}\right]\left[\sum_{\forall m^{\prime} \neq m} \sum_{k \in \mathcal{K}} \beta_{m^{\prime}, k, n_{g}}\right]=0,
$$

$\forall n_{g} \in \mathcal{N}_{g}, \forall g \in \mathcal{G}, \forall m \in \mathcal{M}$. Let $\mathbf{P}=\left[P_{m, k, n_{g}}\right]_{\forall m, g, n_{g}, k}$ and $\boldsymbol{\beta}=\left[\beta_{m, k, n_{g}}\right]_{\forall m, g, n_{g}, k}$ denote the vector of all transmit powers and UAFs of users, respectively. The rate of user $n_{g}$ of BS $m$ in sub-carrier $k$ is

$$
R_{m, k, n_{g}}(\mathbf{P})=\log _{2}\left[1+\frac{P_{m, k, n_{g}} h_{m, k, n_{g}}}{\sigma^{2}+I_{m, k, n_{g}}}\right],
$$

where

$$
I_{m, k, n_{g}}=\sum_{\forall m^{\prime} \in \mathcal{M}, m^{\prime} \neq m} \sum_{\forall g \in \mathcal{G} \forall n_{g}^{\prime} \in \mathcal{N}_{g}, n_{g}^{\prime} \neq n_{g}} P_{m^{\prime}, k, n_{g}^{\prime}} h_{m, k, n_{g}^{\prime}}
$$

and $\sigma^{2}$ is the noise power assumed to be the same for all users in all sub-carriers and BSs. Now, from (1), the required minimum rate of slice $g \in \mathcal{G}$ is

C3 : $\quad \sum_{m \in \mathcal{M}} \sum_{n_{g} \in \mathcal{N}_{g}} \sum_{k \in \mathcal{K}} \beta_{m, k, n_{g}} R_{m, k, n_{g}}(\mathbf{P}) \geq R_{g}^{\mathrm{rvv}}, \quad \forall g \in \mathcal{G}$.

Considering $\mathrm{C} 1-\mathrm{C} 3$, an optimization problem to jointly allocate the BS, sub-carrier and power with the aim of maximizing the energy efficiency can be written as

$$
\min _{\boldsymbol{\beta}, \mathbf{P}} \sum_{m \in \mathcal{M}} \sum_{g \in \mathcal{G}} \sum_{n_{g} \in \mathcal{N}_{g}} \sum_{k \in \mathcal{K}} \beta_{m, k, n_{g}} P_{m, k, n_{g}}
$$

subject to: $\mathrm{C} 1-\mathrm{C} 3$.

The optimization problem (2) has a non-convex objective function due to inter-cell interference and involves non-linear constraints with the combination of continuous and binary variables, i.e., $\mathbf{P}$ and $\boldsymbol{\beta}$. Consequently, (2) is a non-convex mixed-integer, NP-hard optimization problem [10]. Therefore, an efficient algorithm with reasonable computational complexity is needed to solve the resource allocation problem.

\section{Proposed Algorithm}

To solve (2), we propose the iterative Algorithm 1, where first, we calculate $\boldsymbol{\beta}$ based on a given $\mathbf{P}$, and then, use the derived $\boldsymbol{\beta}$ to calculate $\mathbf{P}$ as follows:

$$
\underbrace{\boldsymbol{\beta}(0) \rightarrow \mathbf{P}(0)}_{\text {Initialization }} \rightarrow \ldots \underbrace{\boldsymbol{\beta}(t)^{*} \rightarrow \mathbf{P}(t)^{*}}_{\text {Iteration } t} \rightarrow \underbrace{\boldsymbol{\beta}^{*} \rightarrow \mathbf{P}^{*}}_{\text {Optimal solution }},
$$

where $t>0$ is the iteration number, $\boldsymbol{\beta}(t)^{*}, \mathbf{P}(t)^{*}$ are the optimal values. The iterative procedure is stopped when

$$
\left\|\boldsymbol{\beta}^{*}(t)-\boldsymbol{\beta}^{*}(t-1)\right\| \leq \varepsilon_{1} \text { and }\left\|\mathbf{P}^{*}(t)-\mathbf{P}^{*}(t-1)\right\| \leq \varepsilon_{2}
$$

where $0<\varepsilon_{1} \ll 1$ and $0<\varepsilon_{2} \ll 1$. Note that the user association and power allocation problems are still nonconvex and suffer from high computational complexity. In developing an efficient algorithm, we apply complementary geometric programming (CGP) via different transformations and convexification approaches to solve the sequence of lowerbound GP-based approximations as discussed in the following sub-sections.

\section{A. A brief review of complementary geometric programming}

The standard form of GP is defined as

$$
\begin{array}{cll} 
& \min _{\mathbf{x}} p_{0}(\mathbf{x}), & \\
\text { subject to: } & p_{i}(\mathbf{x}) \leq 1, \quad \forall i=0,1, \ldots, I, \\
& q_{j}(\mathbf{x})=1, \quad \forall j=0,1, \ldots, J,
\end{array}
$$

where $\mathbf{x}=\left[x_{1}, x_{2}, \ldots, x_{N}\right]$ is a non-negative vector of optimization variables, $q_{j}(\mathbf{x})=\prod_{n=1}^{N} c_{j n} x_{n}^{a_{j n}}, p_{i}(\mathbf{x})=$ $\sum_{k=1}^{K_{i}} \prod_{n=1}^{N} c_{i k n} x_{n}^{a_{i k n}}$ for all $i$ are monomial and posynomial functions, respectively where $c_{j n}, c_{i k n}>0, a_{j n}, a_{i k n} \in \Re$. There are restrictions on the constraints in (4) for many practical problems such as (2), e.g., the equality constraints may contain posynomial functions or inequality constraints may not be posynomials. These problems belong to either one of the classes of optimization problems such as complementary geometric programming (CGP) which can be presented as

$$
\begin{array}{ll} 
& \min _{\mathbf{x}} P_{0}(\mathbf{x}), \\
\text { s to: } & P_{i}(\mathbf{x}) \leq 1, \quad \forall i=1, \ldots, I, \\
& Q_{j}(\mathbf{x})=1, \quad \forall j=1, \ldots, J
\end{array}
$$

where $P_{0}(\mathbf{x})=p_{0}^{+}(\mathbf{x}), P_{i}(\mathbf{x})=\frac{p_{i}^{+}(\mathbf{x})}{p_{i}^{-}(\mathbf{x})}, i=1, \cdots, I$ and $Q_{j}(\mathbf{x})=\frac{q_{j}(\mathbf{x})}{p_{j}(\mathbf{x})}$ in which $p_{0}^{+}(\mathbf{x}), p_{0}^{-}(\mathbf{x}), j=0,1, \cdots, J$, are posynomial functions, and $q_{j}(\mathbf{x})$ and $p_{j}(\mathbf{x})$ are monomial and posynomial functions $\forall j$, respectively.

One approach to solve (5) is to convert the CGP into a sequence of standard GP problems [11], [12] that can be solved to reach a global solution. Using the arithmeticgeometric mean approximation (AGMA) to transform the non-posynomial functions to posynomial form, i.e., $P_{i}(\mathbf{x})$, and $Q_{j}(\mathbf{x})$ to its monomial functions, at iteration $l$, the approximated forms of $p_{i}^{-}(\mathbf{x})=\sum_{k=1}^{K_{i}^{-}} q_{k}^{i^{-}}(\mathbf{x})$ and $p_{j}(\mathbf{x})=$ $\sum_{k=1}^{K_{j}} q_{k}^{j}(\mathbf{x})$ are

$$
\widetilde{p}_{i}^{-}(\mathbf{x}(l))=\prod_{k=1}^{K_{i-}}\left(\frac{q_{k}^{i^{-}}(\mathbf{x}(l))}{\alpha_{k}^{i^{-}}(l)}\right)^{\alpha_{k}^{i^{-}}(l)},
$$




$$
\widetilde{p}_{j}(\mathbf{x}(l))=\prod_{k=1}^{K_{j}}\left(\frac{q_{k}^{j}(\mathbf{x}(l))}{\zeta_{k}^{j}(l)}\right)^{\zeta_{k}^{j}(l)}
$$

where $\alpha_{k}^{i^{-}}(l)=\frac{q_{k}^{i^{-}}(\mathbf{x}(l-1))}{p_{i}^{-}(\mathbf{x}(l-1))}$ and $\zeta_{k}^{j}(l)=\frac{q_{k}^{j}(\mathbf{x}(l-1))}{p_{j}(\mathbf{x}(l-1))}$. Now, we have $\widetilde{P}_{i}(\mathbf{x}(l))=\frac{p_{i}^{+}(\mathbf{x}(l))}{\widetilde{p}_{i}^{-}(\mathbf{x}(l))(\mathbf{x}(l))}$ and $\widetilde{Q}_{j}(\mathbf{x}(t))=\frac{q_{j}(\mathbf{x}(t))}{\widetilde{p}_{j}(\mathbf{x}(t))}$ which are posynomial and monomial functions, respectively [11]. Hence, at each iteration $l$, (5) is replaced with

$$
\begin{array}{ll} 
& \min _{\mathbf{x}} P_{0}(\mathbf{x}), \\
\text { s to: } & \widetilde{P}_{i}(\mathbf{x}(l)) \leq 1, \quad \forall i=1, \ldots, I, \\
& \widetilde{Q}_{j}(\mathbf{x}(l))=1, \quad \forall j=1, \ldots, J .
\end{array}
$$

It has been shown that the optimal solution of iterative algorithm from (5) has a very close performance to the optimal solution [11], which can be solved by CVX [9].

\section{B. User-Association Sub-Problem}

For a given $\mathbf{P}(t)$, (2) is transformed into

$$
\min _{\boldsymbol{\beta}} \sum_{m \in \mathcal{M}} \sum_{g \in \mathcal{G}} \sum_{n_{g} \in \mathcal{N}_{g}} \sum_{k \in \mathcal{K}} \beta_{m, k, n_{g}} P_{m, k, n_{g}}(t),
$$

subject to: $\mathrm{C} 1, \mathrm{C} 2, \widetilde{\mathrm{C}} 3$.

Here, for iteration $t, \mathrm{C} 3$ is converted to,

$\widetilde{\mathrm{C}} 3: \sum_{m \in \mathcal{M}} \sum_{n_{g} \in \mathcal{N}_{g}} \sum_{k \in \mathcal{K}} \beta_{m, k, n_{g}} R_{m, k, n_{g}}(\mathbf{P}(t)) \geq R_{g}^{\mathrm{rvv}}, \quad \forall g \in \mathcal{G}$.

In (10), $\boldsymbol{\beta}$ are the optimization (binary) variables. Thus, (10) has much lower computational complexity than (2). Furthermore, we relax $\beta_{m, k, n_{g}} \in[0,1]$, and then use the AGMA to convert $\mathrm{C} 2$ and $\mathrm{C} 3$ as follows. First, we rewrite $\mathrm{C} 3$ as, $\forall g \in \mathcal{G}$,

$$
\widetilde{\mathrm{C}} 3: \frac{R_{g}^{\mathrm{rsv}}}{\sum_{m \in \mathcal{M}} \sum_{n_{g} \in \mathcal{N}_{g}} \sum_{k \in \mathcal{K}} \beta_{m, k, n_{g}} R_{m, k, n_{g}}(\mathbf{P}(t))} \leq 1 .
$$

Now, since the denominator in $\widetilde{\mathrm{C}} 3$ is a posynomial, for each iteration $t_{1}$ of the user association sub-problem, we approximate it and rewrite $\mathrm{C} 3$ as,

$\prod_{m \in \mathcal{M}, n_{g} \in \mathcal{N}_{g}, k \in \mathcal{K}} R_{g}^{\mathrm{rvv}}\left[\frac{\beta_{m k n_{g}}\left(t_{1}\right) R_{m k n_{g}}(\mathbf{P}(t))}{\varphi_{m, k, n_{g}}\left(t_{1}\right)}\right]^{-\varphi_{m, k, n_{g}}\left(t_{1}\right)} \leq 1$ where, $\forall m \in \mathcal{M}, \forall g \in \mathcal{G}, \forall n_{g} \in \mathcal{N}_{G}, \forall k \in \mathcal{K}$,

$$
\begin{aligned}
& \varphi_{m, k, n_{g}}\left(t_{1}\right)= \\
& \frac{\beta_{m, k, n_{g}}\left(t_{1}-1\right) R_{m, k, n_{g}}(\mathbf{P}(t))}{\sum_{m \in \mathcal{M}} \sum_{n_{g} \in \mathcal{N}_{g}} \sum_{k \in \mathcal{K}} \beta_{m, k, n_{g}}\left(t_{1}-1\right) R_{m, k, n_{g}}(\mathbf{P}(t))} .
\end{aligned}
$$

Next, assuming $x_{m, n_{g}}\left(t_{1}\right)=\sum_{k \in \mathcal{K}} \beta_{m, k, n_{g}}\left(t_{1}\right)$ and $y_{n_{g}}\left(t_{1}\right)=\sum_{m \in \mathcal{M}} \sum_{k \in \mathcal{K}} \beta_{m, k, n_{g}}\left(t_{1}\right), \mathrm{C} 2$ can be written as

$$
\begin{array}{r}
x_{m, n_{g}}\left(t_{1}\right)\left[y_{n_{g}}\left(t_{1}\right)-x_{m, n_{g}}\left(t_{1}\right)\right]=0, \\
\forall n_{g} \in \mathcal{N}_{g}, \forall g \in \mathcal{G}, \forall m \in \mathcal{M} .
\end{array}
$$

To convert (12) into a monomial function, we rewrite it as $x_{m, n_{g}}\left(t_{1}\right) y_{n_{g}}\left(t_{1}\right)=x_{m, n_{g}}^{2}\left(t_{1}\right)$, and by adding 1 to both left and right hand sides, we get $x_{m, n_{g}}\left(t_{1}\right) y_{n_{g}}\left(t_{1}\right)+1=1+$ $x_{m, n_{g}}^{2}\left(t_{1}\right)$. Considering $s_{m, n_{g}}\left(t_{1}\right) \geq 0$ as an auxiliary variable,
(12) can be converted into the posynomial inequalities as, [13]

$$
\begin{gathered}
x_{m, n_{g}}\left(t_{1}\right) y_{n_{g}}\left(t_{1}\right)+1 \leq s_{m, n_{g}}\left(t_{1}\right) \leq 1+x_{m, n_{g}}^{2}\left(t_{1}\right), \\
\forall n_{g} \in \mathcal{N}_{g}, \forall g \in \mathcal{G}, \forall m \in \mathcal{M}
\end{gathered}
$$

The above inequalities can be written as

$$
\frac{x_{m, n_{g}}\left(t_{1}\right) y_{n_{g}}\left(t_{1}\right)+1}{s_{m, n_{g}}\left(t_{1}\right)} \leq 1 \text {, and } \frac{s_{m, n_{g}}\left(t_{1}\right)}{1+x_{m, n_{g}}^{2}\left(t_{1}\right)} \leq 1 .
$$

Now, by using AGMA approximation, the above expressions are transformed into

$$
\begin{aligned}
& \text { C2.1: } s_{m, n_{g}}^{-1}\left(t_{1}\right)+x_{m, n_{g}}\left(t_{1}\right) y_{n_{g}}\left(t_{1}\right) s_{m, n_{g}}^{-1}\left(t_{1}\right) \leq 1, \\
& \mathrm{C} 2.2:\left[\frac{1}{\lambda_{m, n_{g}}\left(t_{1}\right)}\right]^{-\lambda_{m, n_{g}}\left(t_{1}\right)} s_{m, n_{g}}\left(t_{1}\right) \times \\
& {\left[\frac{x_{m, n_{g}}^{2}\left(t_{1}\right)}{\alpha_{m, n_{g}}\left(t_{1}\right)}\right]^{-\alpha_{m, n_{g}}\left(t_{1}\right)} \leq 1 \text {, }}
\end{aligned}
$$

where

$$
\begin{aligned}
& \lambda_{m, n_{g}}\left(t_{1}\right)=1 /\left[x_{m, n_{g}}^{2}\left(t_{1}-1\right)+1\right], \\
& \alpha_{m, n_{g}}\left(t_{1}\right)=\frac{x_{m, n_{g}}^{2}\left(t_{1}-1\right)}{x_{m, n_{g}}^{2}\left(t_{1}-1\right)+1} .
\end{aligned}
$$

Now, C2 can be replaced by the following constraints

$$
\mathrm{C} 2.1, \mathrm{C} 2.2 \text {, }
$$

$$
\begin{array}{ll}
\text { C2.3: } & x_{m, n_{g}}\left(t_{1}\right)=\sum_{k \in \mathcal{K}} \beta_{m, k, n_{g}}\left(t_{1}\right), \\
\text { C2.4 : } & y_{n_{g}}\left(t_{1}\right)=\sum_{m \in \mathcal{M}, k \in \mathcal{K}} \beta_{m, k, n_{g}}\left(t_{1}\right) .
\end{array}
$$

Note that via (13), the positive condition for the constraints of GP is met. However, the equality constraints in $\mathrm{C} 2.3$ and $\mathrm{C} 2.4$ are not monomials since we have $x_{m, n_{g}}\left(t_{1}\right)-\sum_{k \in \mathcal{K}} \beta_{m, k, n_{g}}\left(t_{1}\right)=0$ and $y_{n_{g}}\left(t_{1}\right)-$ $\sum_{m \in \mathcal{M}, k \in \mathcal{K}} \beta_{m, k, n_{g}}\left(t_{1}\right)=0$, and, they have negative constraints. To convert $\mathrm{C} 2.3$ and $\mathrm{C} 2.4$ to the monomial functions, we again apply AGMA approximation as

$$
\begin{gathered}
\widetilde{\mathrm{C}} 2.3: x_{m, n_{g}}\left(t_{1}\right) \prod_{k \in K}\left[\frac{\beta_{m, k, n_{g}}\left(t_{1}\right)}{\nu_{m, k, n_{g}}\left(t_{1}\right)}\right]^{-\nu_{m, k, n_{g}}\left(t_{1}\right)}=1, \\
\widetilde{\mathrm{C}} 2.4: y_{n_{g}}\left(t_{1}\right) \prod_{m \in \mathcal{M}, k \in \mathcal{K}}\left[\frac{\beta_{m, k, n_{g}}\left(t_{1}\right)}{\eta_{m, k, n_{g}}\left(t_{1}\right)}\right]^{-\eta_{m, k, n_{g}}\left(t_{1}\right)}=1,
\end{gathered}
$$

where $\nu_{m, k, n_{g}}\left(t_{1}\right)$ and $\eta_{m, k, n_{g}}\left(t_{1}\right)$ are given by,

$$
\begin{aligned}
\nu_{m, k, n_{g}}\left(t_{1}\right) & =\frac{\beta_{m, k, n_{g}}\left(t_{1}-1\right)}{\sum_{k \in \mathcal{K}} \beta_{m, k, n_{g}}\left(t_{1}-1\right)}, \\
\eta_{m, k, n_{g}}\left(t_{1}\right) & =\frac{\beta_{m, k, n_{g}}\left(t_{1}-1\right)}{\sum_{m \in \mathcal{M}} \sum_{k \in \mathcal{K}} \beta_{m, k, n_{g}}\left(t_{1}-1\right)} .
\end{aligned}
$$

Hence, the problem for sub-problem 1 can be written as

$$
\min _{\boldsymbol{\beta}\left(\boldsymbol{t}_{1}\right)} \sum_{m \in \mathcal{M}} \sum_{g \in \mathcal{G}} \sum_{n_{g} \in \mathcal{N}_{g}} \sum_{k \in \mathcal{K}} \beta_{m, k, n_{g}}\left(t_{1}\right) P_{m, k, n_{g}}(t),
$$

s.t: $\mathrm{C} 1, \mathrm{C} 2.1-\mathrm{C} 2.2, \widetilde{\mathrm{C}} 2.3-\widetilde{\mathrm{C}} 2.4, \widetilde{\mathrm{C}} 3$. 
Now, (17) belongs to GP and can be solved using CVX.

\section{Power Minimization Sub-Problem}

For given $\boldsymbol{\beta}$, the power allocation problem is

$$
\min _{\mathbf{P}\left(t_{2}\right)} \sum_{m \in \mathcal{M}} \sum_{g \in \mathcal{G}} \sum_{n_{g} \in \mathcal{N}_{g}} \sum_{k \in \mathcal{K}} \beta_{m, k, n_{g}}(t) P_{m, k, n_{g}}\left(t_{2}\right)
$$

subject to:

$$
\widetilde{\mathrm{C}} 3: \sum_{m \in \mathcal{M}} \sum_{k \in \mathcal{K}} \sum_{n_{g} \in \mathcal{N}_{g}} \beta_{m, k, n_{g}}(t) R_{m, k, n_{g}}\left(\mathbf{P}\left(t_{2}\right)\right) \geq R_{g}^{\mathrm{rsv}},
$$

where $t_{2}$ is the iteration index. Due to interference in the objective function of $R_{m, k, n_{g}}\left(\mathbf{P}\left(t_{2}\right)\right)$, (18) is a non-convex optimization problem. We again follow the AGMA approach to convert (18) into the equivalent GP problem. First, we rewrite $\widetilde{\mathrm{C}} 3$ as

$$
\prod_{m \in \mathcal{M}, n_{g} \in \mathcal{N}_{g}, k \in \mathcal{K}} \gamma_{m, k, n_{g}}\left(\mathbf{P}\left(t_{2}\right)\right) \leq 2^{-R_{g}^{\mathrm{rsv}}}, \quad \forall g \in \mathcal{G},
$$

where

$$
\gamma_{m, k, n_{g}}\left(\mathbf{P}\left(t_{2}\right)\right)=\frac{\sigma^{2}+I_{m, k, n_{g}}\left(t_{2}\right)+P_{m, k, n_{g}}\left(t_{2}\right) h_{m, k, n_{g}}}{\sigma^{2}+I_{m, k, n_{g}}\left(t_{2}\right)},
$$

and

$$
\sum_{\forall m^{\prime} \neq m}^{I_{m, k, n_{g}}\left(t_{2}\right)=} \sum_{\forall g} \sum_{\forall n_{g}^{\prime} \neq n_{g}} P_{m^{\prime}, k, n_{g}^{\prime}}\left(t_{2}\right) h_{m, k, n_{g}^{\prime}} .
$$

Now using AGMA approach, $\gamma_{m, k, n_{g}}\left(\mathbf{P}\left(t_{2}\right)\right)$ can be approximated as

$$
\begin{aligned}
& \widehat{\gamma}_{m, k, n_{g}}\left(\mathbf{P}\left(t_{2}\right)\right)=\left(\sigma^{2}+I_{m, k, n_{g}}\left(t_{2}\right)\right)\left(\frac{\sigma^{2}}{\kappa_{o}\left(t_{2}\right)}\right)^{-\kappa_{o}\left(t_{2}\right)} \\
& \times \prod_{m \in \mathcal{M}, n_{g} \in \mathcal{N}_{g}, k \in \mathcal{K}}\left(\frac{P_{m, k, n_{g}}\left(t_{2}\right) h_{m, k, n_{g}}}{\kappa_{m, k, n_{g}}\left(t_{2}\right)}\right)^{-\kappa_{m, k, n_{g}}\left(t_{2}\right)},
\end{aligned}
$$

where

$$
\begin{gathered}
\kappa_{m, k, n_{g}}\left(t_{2}\right)= \\
\frac{P_{m, k, n_{g}}\left(t_{2}-1\right) h_{m, k, n_{g}}}{\sigma^{2}+\sum_{m \in \mathcal{M}, n_{g} \in \mathcal{N}_{g}, g \in \mathcal{G}} P_{m, k, n_{g}}\left(t_{2}-1\right) h_{m, k, n_{g}}}, \\
\kappa_{o}\left(t_{2}\right)=\frac{\sigma^{2}}{\sigma^{2}+\sum_{m \in \mathcal{M}, n_{g} \in \mathcal{N}_{g}, g \in \mathcal{G}} P_{m, k, n_{g}}\left(t_{2}-1\right) h_{m, k, n_{g}}} .
\end{gathered}
$$

Consequently, (18) is transformed into

$$
\min _{\mathbf{P}\left(t_{2}\right)} \sum_{m \in \mathcal{M}} \sum_{g \in \mathcal{G}} \sum_{n_{g} \in \mathcal{N}_{g}} \sum_{k \in \mathcal{K}} \beta_{m, k, n_{g}}(t) P_{m, k, n_{g}}\left(t_{2}\right)
$$

subject to:

$$
\text { C3 : } \prod_{m \in \mathcal{M}, g \in \mathcal{G}, n_{g} \in \mathcal{N}_{g}, k \in \mathcal{K}} \widehat{\gamma}_{m, k, n_{g}}\left(\mathbf{P}\left(t_{2}\right)\right) \leq 2^{-R_{g}^{\mathrm{rvv}}}, \quad \forall g \in \mathcal{G} \text {. }
$$

The overall optimization problem is iteratively solved as described in Algorithm 1 until the UAF and the power vector converges, i.e., $\left\|\boldsymbol{\beta}\left(t_{1}\right)-\boldsymbol{\beta}\left(t_{1}-1\right)\right\| \leq \varepsilon_{1}$ and $\| \mathbf{P}\left(t_{2}\right)-\mathbf{P}\left(t_{2}-\right.$ $1) \| \leq \varepsilon_{2}$ where $0<\varepsilon_{1}, \varepsilon_{2} \ll 1$.

\begin{abstract}
Algorithm 1:
Initialization: Set $t=t_{1}=t_{2}=1, \boldsymbol{\beta}(t)=[\mathbf{1}]$, where $\mathbf{1}$ is a vector $C^{1 \times K N}$ and $\mathbf{P}(t)$ with power of each sub-carrier of BS $m$ as $P_{m}^{\max } / K$.
\end{abstract}

\section{Repeat:}

Step 1: Repeat: Set $\boldsymbol{\beta}\left(t_{1}=1\right)=\boldsymbol{\beta}(t), \mathbf{P}\left(t_{1}=1\right)=\mathbf{P}(t)$ and set arbitrary initial for $s_{m, n_{g}}\left(t_{1}\right)$,

Step 1a: Update $\lambda_{m, n_{g}}\left(t_{1}\right), \alpha_{m, n_{g}}\left(t_{1}\right), \nu_{m, k, n_{g}}\left(t_{1}\right)$, $\eta_{m, k, n_{g}}\left(t_{1}\right)$ and $\varphi_{m, k, n_{g}}\left(t_{1}\right)$ as per (11) - (16),

Step 1b: Find optimal UAF in (17) using CVX [9],

Until $\left\|\boldsymbol{\beta}^{*}\left(t_{1}\right)-\boldsymbol{\beta}^{*}\left(t_{1}-1\right)\right\| \leq \varepsilon_{1}$.

Step 2: Repeat: Obtain $\boldsymbol{\beta}\left(t_{2}=1\right)=\boldsymbol{\beta}^{*}\left(t_{1}\right)$.

Step 2a: Update $\kappa_{m, k, n_{g}}\left(t_{2}\right)$ and $\kappa_{o}\left(t_{2}\right)$ from (20),

Step 2b: Find optimum power from (21) via CVX [9],

Until $\left\|\mathbf{P}\left(t_{2}\right)-\mathbf{P}\left(t_{2}-1\right)\right\| \ll \varepsilon_{2}$.

$\underline{\text { Until: }\left\|\boldsymbol{\beta}^{*}(t)-\boldsymbol{\beta}^{*}(t-1)\right\| \leq \varepsilon_{1} \text {, and }\left\|\mathbf{P}^{*}(t)-\mathbf{P}^{*}(t-1)\right\| \leq \varepsilon_{2} .}$

\section{Simulation Results}

We consider a multi-cell VWN with $M=4 \mathrm{BSs}$, each with a radius of $500 \mathrm{~m}, G=2, K=4$ and $N=8$ users uniformly distributed within the area. The channel gains are derived from the Rayleigh fading model as $h_{m, n_{g}, k}=\chi_{m, n_{g}, k} d_{m, n_{g}}^{-\zeta}$ where $\zeta=3$ is the path loss exponent, $d_{m, n_{g}}$ is the distance between the BS $m$ and user $n_{g}$ and $\chi_{m, n_{g}, k}$ is the exponential random variable with mean of 1 . For all of the simulations, we set $\varepsilon_{1}=10^{-5}$ and $\varepsilon_{2}=10^{-6}$, and $R^{\text {rsv }}=R_{g}^{\text {rsv }}$ for all $g \in \mathcal{G}$ unless otherwise stated. The results are demonstrated based on the average over 100 channel realizations. To compare the performance of our algorithm, we use the sub-optimal approach where the users are assigned to BS based on the received signal strength. Hence, here, the problem is

$$
\min _{\boldsymbol{\beta}, \mathbf{P}} \sum_{m \in \mathcal{M}} \sum_{g \in \mathcal{G}} \sum_{n_{g} \in \mathcal{N}_{g}} \sum_{k \in \mathcal{K}} \beta_{m, k, n_{g}} P_{m, k, n_{g}},
$$

subject to: $\mathrm{C} 1, \mathrm{C} 3$.

The proposed Algorithm 1 can be applied for solving (22) except that all constraints related to $\mathrm{C} 2$ are removed since the $\mathrm{BS}$ assignment is predetermined based on the received signal strength.

Fig. 1 shows the total transmit power of all BSs versus $R_{g}^{\text {rsv }}$ and $K$ for both Alg. 1 and the sub-optimal approach. As expected, due to the opportunistic nature of fading channels [14], by increasing $K$, the total transmit power decreases. However, the total transmit power required by the sub-optimal approach is significantly higher than the one required by Alg. 1. This is because the BS assignment is based on the highest received signal strength in the sub-optimal approach. Therefore, more transmit power is required to support the rate reservation per slice. Also, with increased rate reserved per slice, the total transmit power increases since the BSs need to transmit at a higher power to support the reserved rate.

Fig. 2 plots the total transmit power of all BSs versus $N$ for different $R^{\text {rsv }}$. Clearly, the total transmit power decreases with increasing $N$ for a fixed $R^{\text {rsv }}$ because, from the user diversity 


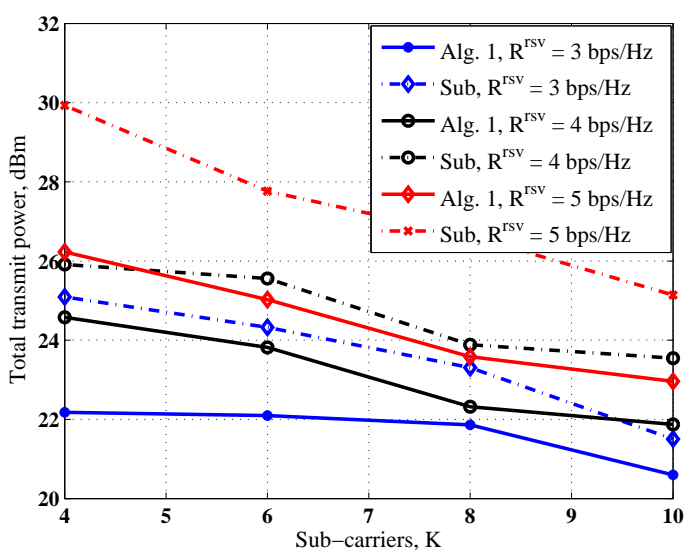

Fig. 1. Total transmit power versus $K$

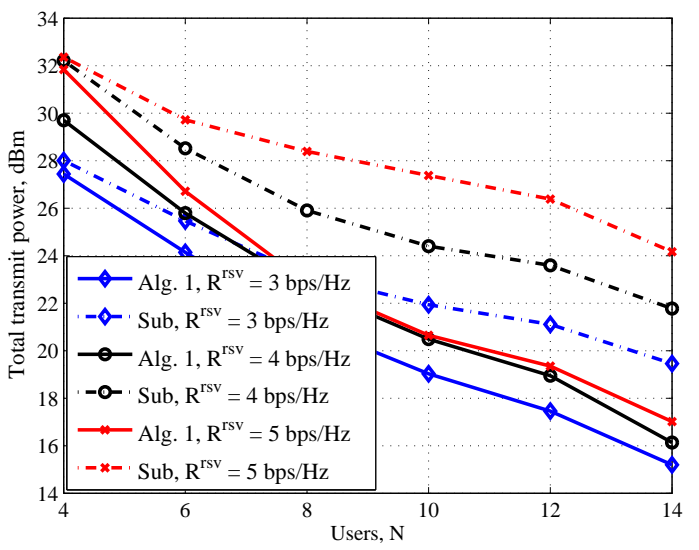

Fig. 2. Total transmit power versus $N$.

gain, there is a higher chance of assigning the sub-carriers with less power while fulfilling the minimum rate constraint per slice. Also, similar to Fig. 1, the total transmit power of the sub-optimal approach is higher than that of Alg. 1 with the difference becoming more distinguishable at higher values of $N$. Moreover, the total transmit power increases with the increase in $R^{\mathrm{rsv}}$.

In Fig. 3, the effects of increasing the number of BSs, i.e., $M$, is investigated with $N=32$. It can be observed that increasing $M$ does not have monotonic effect on the transmit power. For instance, for $R^{\text {rsv }}=3$ and $R^{\text {rsv }}=4$, the total required power at $M=4$ is lower than that for $M=9$ and 16. It is mainly because the inter-cell interference will be increased by increasing the number of BSs, which results in higher power requirement. However, large value of $R^{\text {rsv }}$ such as $R^{\mathrm{rsv}}=5 \mathrm{bps} / \mathrm{Hz}$, cannot be attained by $M=4$. Therefore, the number of BSs should be properly designed for a specific region to reach to an energy efficient and feasible VWN. Further study of this trade-off remains as a future work.

\section{CONCLUSION}

In this paper, we consider an energy-efficient resource allocation in an OFDMA multi-cell VWN where users belonging to different slices require a diverse minimum rate reservation.

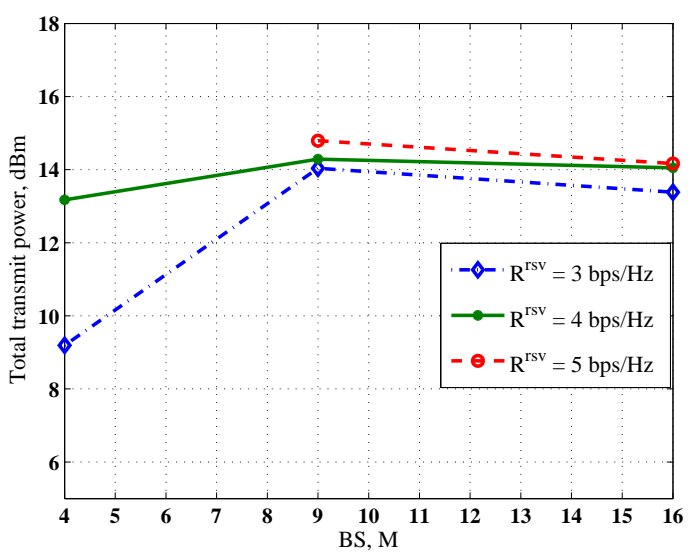

Fig. 3. Total transmit power versus $M$

We propose a novel constraint for the joint BS and subcarrier allocation and convert the highly complex optimization problem into more tractable formulation via CGP and SCA. Simulation results reveal the performance gains of the proposed algorithm with respect to different system parameters and indicate the importance of efficient BS allocation to improve energy efficiency.

\section{REFERENCES}

[1] R. Kokku, R. Mahindra, H. Zhang, and S. Rangarajan, "NVS: A substrate for virtualizing wireless resources in cellular networks," IEEE/ACM Trans. Netw., vol. 20, no. 5, pp. 1333-1346, Oct. 2012.

[2] K. Tan, H. Shen, J. Zhang, and Y. Zhang, "Enabling flexible spectrum access with spectrum virtualization," in IEEE International Symposium on Dynamic Spectrum Access Networks (DYSPAN), Oct. 2012.

[3] C. Liang and F. Yu, "Wireless network virtualization: A survey, some research issues and challenges," IEEE Commun. Surveys Tuts., vol. 17, no. 1 , pp. $358-380,2014$.

[4] F. Fu and U. Kozat, "Stochastic game for Wireless Network Virtualization," IEEE/ACM Trans. Netw., vol. 21, no. 1, pp. 84-97, Feb. 2013.

[5] S. Parsaeefard, V. Jumba, M. Derakhshani, and T. Le-Ngoc, "Joint Resource Provisioning and Admission Control in Wireless Virtualized Networks," in Proc. IEEE Wireless Commun. Netw. Conf. (WCNC), Mar. 2015, pp. $2020-2025$.

[6] M. Yang, Y. Li, D. Jin, J. Yuan, I. You, and L. Zeng, "Opportunistic sharing scheme for spectrum allocation in Wireless Virtualization," IEEE Trans. Services Comput., vol. 18, no. 9, pp. 1685-1696, 2014.

[7] G. Liu, F. Yu, H. Ji, and V. Leung, "Distributed resource allocation in full-duplex relaying networks with wireless virtualization," in Proc. IEEE Global Commun. Conf. (GLOBECOM), Dec. 2014.

[8] V. Jumba, S. Parsaeefard, M. Derakhshani, and T. Le-Ngoc, "Resource Provisioning in Wireless Virtualized Networks via massive-MIMO," IEEE Commun. Lett., vol. 4, no. 3, Feb. 2015.

[9] M. Grant and S. Boyd, "CVX: Matlab software for disciplined convex programming, version 2.1," http://cvxr.com/cvx, 2014.

[10] Z.-Q. Luo and S. Zhang, "Dynamic spectrum management: Complexity and duality," IEEE J. Sel. Topics Signal Process., vol. 2, no. 1, pp. 5773, Feb. 2008.

[11] G. Xu, "Global optimization of signomial geometric programming problems," European Journal of Operational Research, vol. 233, no. 3, pp. 500-510, 2014.

[12] M. Derakhshani, X. Wang, T. Le-Ngoc, and A. Leon-Garcia, "Virtualization of multi-cell 802.11 networks: Association and airtime control," IEEE Trans. Mobile Comput., submitted Aug. 2015, arXiv preprint arXiv: 1508.03554.

[13] M. Chiang, "Geometric Programming for communication systems," Foundations and Trends in Communications and Information Theory, vol. 2, no. 1-2, pp. 1-154, 2005.

[14] A. Goldsmith, Wireless Comunications. Cambridge University Press, 2004. 